\title{
Optogenetic activation of dorsal raphe neurons rescues the autistic-like social deficits in Shank3 knockout mice
}

\author{
Cell Research (2017) 27:950-953. doi:10.1038/cr.2017.52; published online 4 April 2017
}

\section{Dear Editor,}

Impaired social interaction is one of the core symptoms of the autism spectrum disorder (ASD) [1]. However, the etiology and neural circuit mechanisms underlying these behavioral impairments are not well understood [2]. Currently, behavioral therapies are the most effective interventions for ASD, although the benefits from such treatments are minimal $[3,4]$. Here, we demonstrate that the autistic-like social deficits of Shank3 knockout mice [5] can be durably rescued by social training coupled with optogenetic activation of neurons in the midbrain dorsal raphe nucleus (DRN) - a region encoding reward through 5-HT and glutamate [6]. A single training session resulted in a significant rescue effect on social preference, and such rescue effect was retained for several days with increased neuronal activation in the DRN when facing social stimuli. Multiple training sessions resulted in much longer-lasting rescue effects. Intriguingly, the durable rescue effect was generated by stimulating Pet-1 neurons in the DRN, but not by stimulating dopamine neurons in the ventral tegmental area (VTA) - the classical reward center. Our results suggest that the DRN should be viewed as an attractive target for future ASD interventions.

The social motivation hypothesis proposes that social impairments result from attributing decreased level of reward to social interaction, a process that has been putatively associated with abnormal brain functions in individuals with ASD [4]. Functional neuroimaging studies have indicated that social deficits in ASD are related to dysfunction of the reward circuitry in the brain [7]. Optogenetic approaches have been widely used to study the neural circuits underlying social behaviors [8-10]. Based on recent findings that optogenetic activation of Pet1 neurons in the DRN [6] or dopamine neurons in the VTA [10-12] can produce strong reward signals and reinforce mouse behaviors, we tested whether such activation may be effective in rescuing the autistic-like social deficits in Shank3 mutant autism mouse model [5]. We crossed Shank $3^{--}$mice with the ePet1-Cre mouse line [13] or the DAT-Cre mouse line [14]. A Cre-dependent AAV viral vector was then used to achieve the cell type- and brain region-specific (DRN or VTA) expression of either ChR2-mCherry or mCherry. Optical fibers were subsequently implanted at the virus-injection sites. Only male animals were used across all the behavior tests.

Social training coupled with optogenetic activation of DRN was performed on the following animal groups: Shank $3^{+/+}$: ePet1-Cre-DRN ${ }^{\text {mCherry }}$ mice (wild-type, WT); Shank $^{-1-}$ : ePet1-Cre-DRN ${ }^{\text {mCherry }}$ mice (knockout, KO); Shank3 $3^{+++}$: ePet1-Cre-DRN ${ }^{\text {ChR2-mCherry }}$ mice (rewarded-WT); Shank3 ${ }^{-/}$: ePet1-Cre-DRN ${ }^{\text {ChR2-mCherry }}$ mice (rewarded-KO; Figure 1A, 1B and Supplementary information, Figure S1). The social training was performed in a rectangular chamber that contained two cages: one empty cage and one cage with a stranger mouse inside (termed the social stimulus). Test mice were allowed to move freely and were monitored in real-time. Cranial light pulses to the DRN were triggered when test mice interacted closely with the social stimulus (within $8 \mathrm{~cm}$ radius), and were ceased immediately when mice left the proximity of the stimulus. Unlike WT mice, the KO mice showed no preference for the social stimulus, implying autistic-like social deficits. Conversely, the rewarded-KO mice exhibited a strong preference for the social stimulus which was even stronger than that of the WT mice (Figure 1C). After the training session, we immediately switched the positions of the two cages and monitored the movement of the test mice without delivering light pulses for an additional $10 \mathrm{~min}$, to exclude the possibility of any positional preferences. Data from the post-training periods showed that the rewarded-KO mice retained their preference for the social stimulus (Figure 1D), indicating that the social-light coupling led to short-term social learning.

We next used the standard three-chamber assay to evaluate the sociability of these mice three days after the initial social training experiments. Consistent with findings from the initial social training sessions, the KO mice displayed no preference for the social stimulus three days after the end of the social training. Strikingly, the 
A

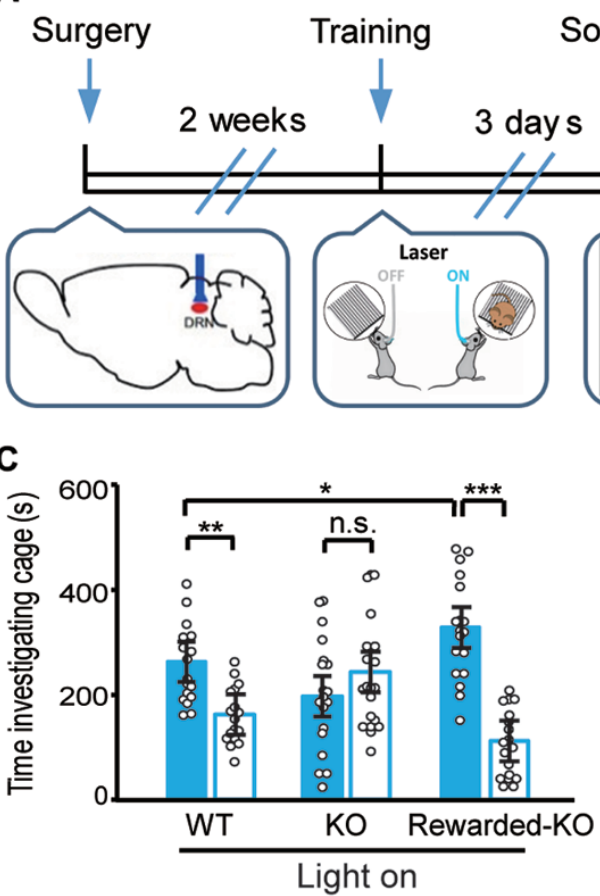

E

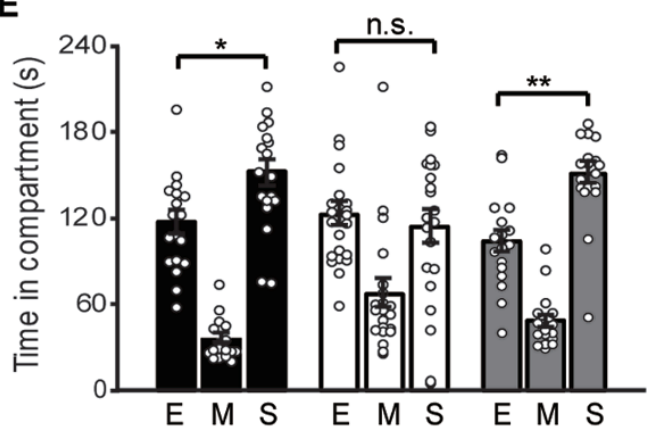

D
Social evaluation
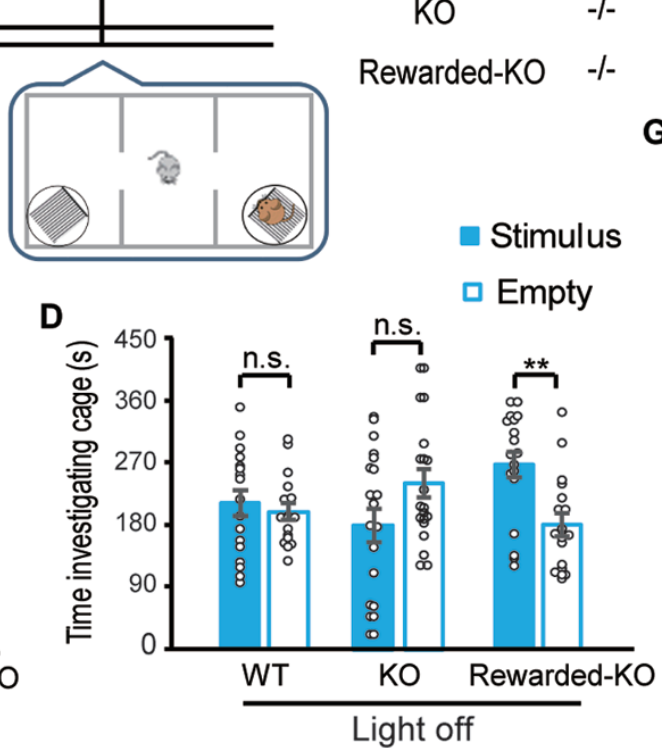

$F$

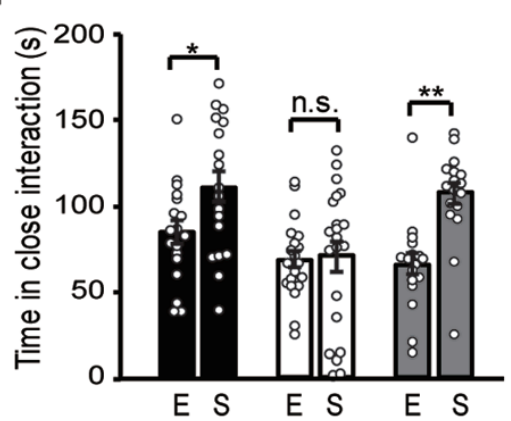

E- Empty M- Middle S - Stranger mouse

$M$

Stimulus

Empty

B
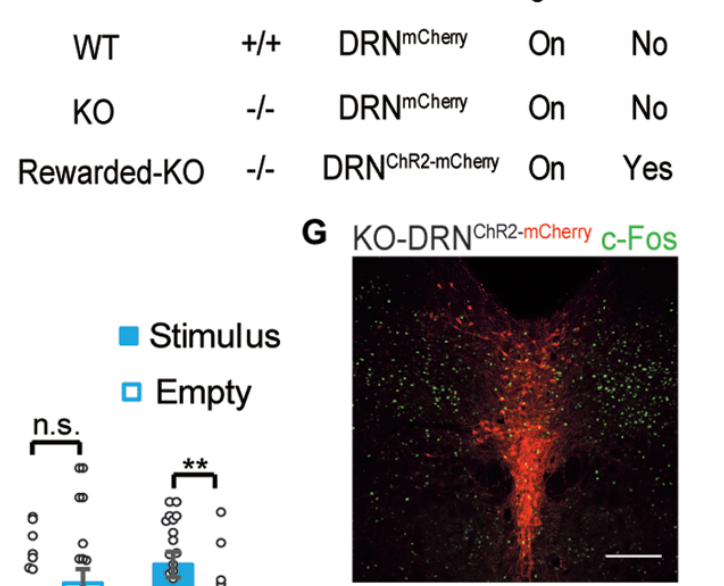

KO-DRN ${ }^{\text {mCherry }}$ c-Fos

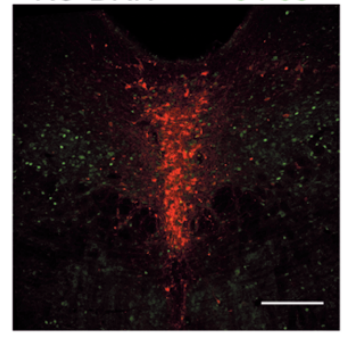

H $\square$ mCherry+ $\square$ Total
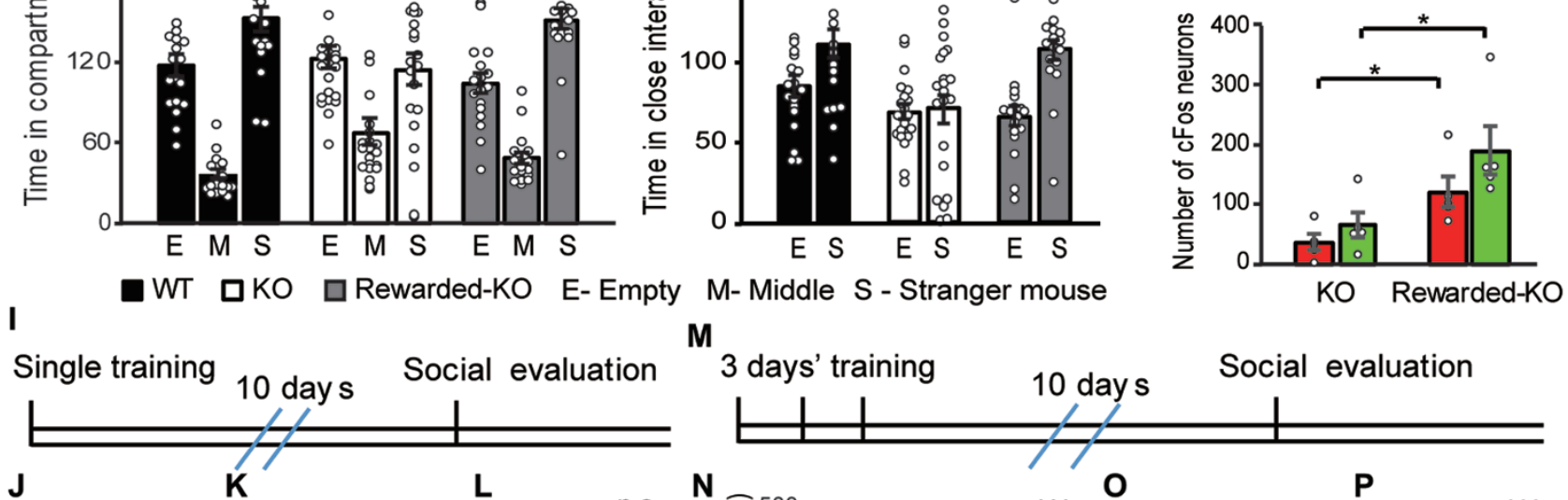

3 days' training

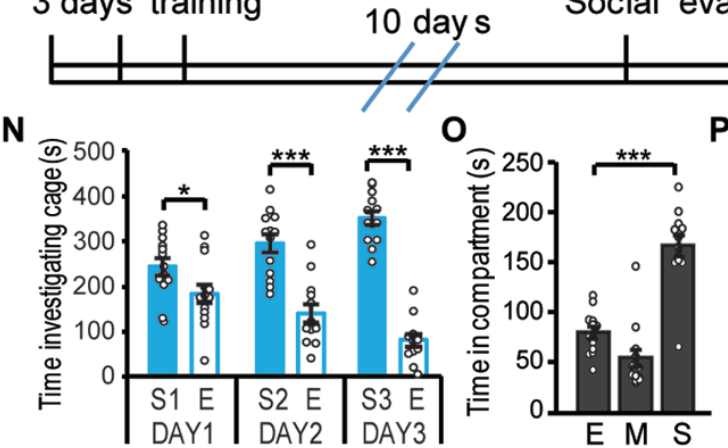

10 days

Social evaluation
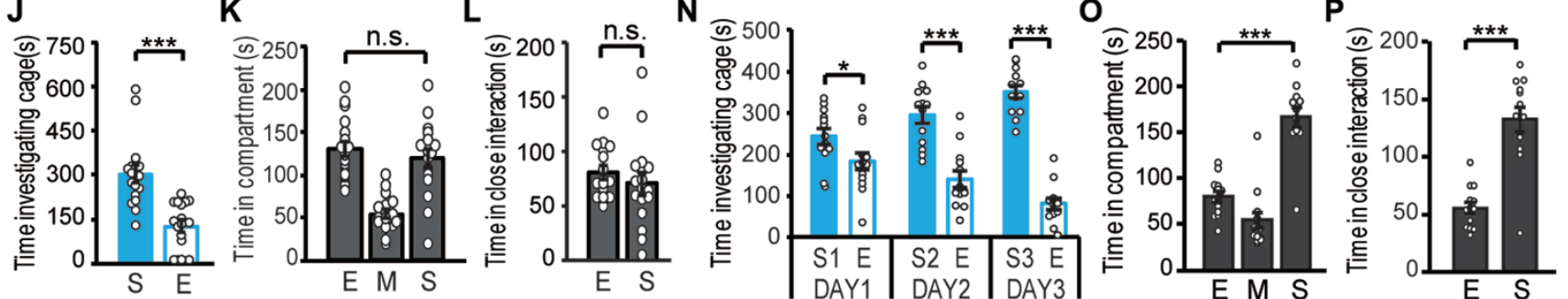

Figure 1 Coupling social interaction with optogenetic activation of DRN Pet-1 neurons rescues the social deficit of Shank3 knockout mice. (A) Timeline of the experimental procedures. ChR2 was selectively expressed in the DRN of ePet1-Cre mice (lower left). Cranial light pulses (blue) were triggered only when test mice were in proximity to the cage that contained a mouse as the social stimulus (lower middle). A three-chamber test evaluated sociability after training (lower right). (B) Detailed information of the three animal groups ( $n=18$ for WT, 21 for KO, 18 for rewarded-KO). (C, D) Scatter plots and group means of time spent investigating the social stimulus or the empty cage during a 10 min training session (C) and a 10 min post-training session (D). (E, F) Scatter plots and group means of time spent in each compartment (E) and in close interaction with each cage (F) in the three-chamber test. (G) Distribution of social interaction-induced c-Fos ${ }^{+}$cells in the DRN of Shank3 
rewarded-KO mice still showed a strong preference for the social stimulus as compared to the empty cage; such preference was also observed in both WT (Figure 1E and $1 \mathrm{~F}$ ) and the rewarded-WT mice (Supplementary information, Figure S1J and S1K). There is no significant difference between the sociability of the WT group and the serotonin rewarded-WT group, suggesting that the effect of DRN stimulation on social behavior became evident only in socially impaired animals. To confirm that the rescued social preference resulted from social training with the real social stimulus, rather than from a neutral object, we carried out the same training procedure on another group of rewarded-KO mice using an inanimate toy mouse instead of the real stranger mouse during training and, three days later, tested their preference towards a stranger mouse and an inanimate toy mouse in the three-chamber assay. Although optogenetic stimulation during the initial training induced a strong preference for the toy mouse (Supplementary information, Figure S2A-S2D), the preference failed to be transfered to the social stimulus three days later in the three-chamber assay (Supplementary information, Figure S2E and S2F). Thus, the social stimulus (i.e., a real mouse rather than an object) is an indispensable component of the social training process. The results from these training and social evaluation assays demonstrate that social deficits can be rescued by social training coupled with optogenetic activation of the DRN, and most surprisingly of all, the rescue effect can persist for several days.

To determine whether social training results in changes in brain activity, we measured the expression level of c-Fos in the DRN induced by home-cage social interaction three days after the training. We observed obviously increased c-Fos expression in the DRN of the rewarded-KO mice as compared to the KO mice (Figure 1G and $1 \mathrm{H}$ ). These results, viewed alongside our behavioral results, indicate that the training may endow the autistic mice with the ability to self-activate neurons in the DRN during social interactions, thereby providing reward for increased social behavior.

To evaluate if the rescue effect could persist for even longer periods, we trained another group of rewarded-KO mice and tested sociability after ten days (Figure 1I). The social preference exhibited during the initial training (Figure $1 \mathrm{~J}$ ) was not observed ten days later (Figure $1 \mathrm{~K}$ and $1 \mathrm{~L}$ ). We then modified the experimental design by conducting training sessions on three consecutive days and testing sociability ten days after the final training session (Figure 1M). Encouragingly, the rewarded-KO mice that received multiple trainings (Figure 1N) showed a strong social preference ten days after training (Figure $1 \mathrm{O}$ and $1 \mathrm{P}$ ), indicating that multiple trainings can significantly extend the rescue effect.

We also tested whether the coupling of social training with optogenetic activation of dopamine neurons in the VTA of DAT-Cre mice could rescue the social deficits of Shank3 knockout mice on the following four animal groups: Shank3 $3^{++}$: DAT-Cre-VTA ${ }^{\text {mCherry }}$ mice (WT); Shank $^{-1-}$ : DAT-Cre-VTA ${ }^{\text {mCherry }}$ mice (KO); DAT-Cre$\mathrm{VTA}^{\text {ChR2-mCherry }}$ mice (rewarded-WT); Shank $3^{-/}$: DATCre-VTA ${ }^{\text {ChR2-mCherry }}$ mice (rewarded-KO) (Supplementary Information, Figure S3A and S3B). Consistent with the recent study on dopamine stimulation [12], coupling optogenetic stimulation of dopamine neurons with social interaction produced strong preferences for the social stimulus over the empty cage in both Shank3 intact and Shank3 mutant mice (Supplementary information, Figure $\mathrm{S} 3 \mathrm{C}$ ). However, when the positions of the cages were switched and no light pulses were delivered during the test sessions, both rewarded-WT and rewarded-KO animals lost the stimulation-induced social preference (Supplementary information, Figure S3D). In three-chamber sociability assays, neither the KO mice nor the rewarded-KO mice, as compared to the WT mice, showed preference for the social stimulus (Supplementary information, Figure S3E and S3F). Thus, social training coupled

knockout mice with (upper) or without (lower) light-social coupling. Red: Pet1-mCherry; Green: c-Fos. Scale bar, $200 \mu \mathrm{m}$. (H) Quantitation of c-Fos ${ }^{+}$neurons in mCherry-expressing area (red) and in the entire DRN (green) of the two groups of Shank3 mutant mice ( $n=5$ mice for each group). Light-social coupling resulted in significantly higher c-Fos induction. (I) Schematic of the single training experimental procedure. Shank3 knockout mice underwent one training session and were tested for sociability 10 days later $(n=17$ mice). (J) Scatter plots and group means of time spent investigating the social stimulus or the empty cage during a 10 min training session. (K, L) Scatter plots and group means of time spent in each compartment (K) and in close interaction with each cage (L) in the three-chamber test 10 days after the single training session. (M) Schematic of the multiple training experimental procedure. Shank3 knockout mice underwent three training sessions (10 min per day) and were tested for sociability 10 days later $(n=13$ mice). (N) Scatter plots and group means of time spent investigating the social stimuli or the empty cage in a 10 min training session over three consecutive days. S1/2/3: Three age-matched stranger mice. $(\mathbf{O}, \mathbf{P})$ Scatter plots and group means of time spent in each compartment $(\mathbf{O})$ and in close interaction with each cage $(\mathbf{P})$ in the three-chamber test 10 days after completing the three training sessions. ${ }^{*} P<0.05$; ${ }^{* *} P<0.01$; ${ }^{* * *} P<0.001$; one-way ANOVA followed by Bonferroni post hoc $t$-tests and Kruskal-Wallis rank sum tests for $\mathbf{C}, \mathbf{D}, \mathbf{E}$ and $\mathbf{F}$. Wilcoxon signed rank test for $\mathbf{H}, \mathbf{J}$ and $\mathbf{P}$; one-tailed paired $t$-test for $\mathbf{K}$, $\mathbf{L}$ and $\mathbf{O}$; Repeated measures two-way ANOVA for $\mathbf{N}$. Error bars show SEM. 
with optogenetic activation of dopamine neurons in the VTA is insufficient to rescue the dysfunctional social behavior phenotype of the Shank3 knockout mice.

In summary, we found that the autistic-like social deficits in Shank3 knockout mice can be durably rescued via social training coupled with optogenetic activation of Pet1 neurons in the DRN. Such training could possibly endow the rescued mice with the ability to self-activate neurons in the DRN during social interactions, thus positively reinforcing social behavior. Multiple training sessions significantly reinforced the social learning, and resulted in more durable rescue effects. Previous studies showed that optogenetic activation of dopamine neurons in the VTA increased the social preference $[9,12]$. However, our results showed that such social preference only appears during the light-social stimulus coupled session, and does not result in a durable rescue effect as compared to Pet1 neuron activation. Pet-1 neurons in the DRN release 5-HT and glutamate [6], suggesting the specific role of social rewards encoded by DRN through 5-HT and glutamate in guiding social behavior. Therefore, the DRN should be viewed as a potentially important target for the development of therapies targeting social deficits in ASD.

\section{Acknowledgments}

We thank Y Li, Z Liu and R Lin in the Luo Lab and Y Ye and S An in the Wei Lab for technical assistance, and JH Snyder for manuscript editing. ML is supported by Ministry of Science and Technology of China (2012YQ03026005, 2013ZX0950910, 2015BAI08B02), National Natural Science Foundation of China
(91432114, 91632302), and the Beijing Municipal Government.

\author{
Junyu Luo ${ }^{1}$, Qiru Feng ${ }^{1}$, Liping Wei ${ }^{2,3}$, Minmin Luo ${ }^{1,3}$ \\ ${ }^{I}$ Peking University-Tsinghua University-National Institute of Biological \\ Sciences (PTN) Joint Graduate Program, School of Life Sciences, \\ Tsinghua University, Beijing 100084, China; ${ }^{2}$ School of Life Sciences, \\ Peking University, Beijing 100871, China: ${ }^{3}$ National Institute of Biological \\ Sciences, Beijing 102206, China \\ Correspondence: Liping $\mathrm{Wei}^{\mathrm{a}}, \mathrm{Minmin} \mathrm{Luo}^{\mathrm{b}}$ \\ ${ }^{\mathrm{a}}$ Tel: 010-80723791 \\ E-mail: weilp@mail.cbi.pku.edu.cn \\ bE-mail: luominmin@nibs.ac.cn
}

\section{References}

1 Association AP. Diagnostic and Statistical Manual of Mental Disorders. 5th Edition. Washington, DC: American Psychiatric Association, 2013.

2 Won H, Mah W, Kim E. Front Mol Neurosci 2013; 6:19.

3 Granpeesheh D, Tarbox J, Dixon DR. Ann Clin Psychiatry 2009; 21:162-173.

4 Dawson G. Dev Psychopathol 2008; 20:775-803.

5 Peca J, Feliciano C, Ting JT, et al. Nature 2011; 472:437-442.

6 Liu Z, Zhou J, Li Y, et al. Neuron 2014; 81:1360-1374.

7 Kohls G, Schulte-Ruther M, Nehrkorn B, et al. Soc Cogn Affect Neurosci 2013; 8:565-572.

8 Boyden ES, Zhang F, Bamberg E, et al. Nat Neurosci 2005; 8:12631268.

9 Gunaydin LA, Grosenick L, Finkelstein JC, et al. Cell 2014; 157:1535-1551.

10 Keiflin R, Janak PH. Neuron 2015; 88:247-263.

11 Wise RA, Rompre PP. Annu Rev Psychol 1989; 40:191-225.

12 Bariselli S, Tzanoulinou S, Glangetas C, et al. Nat Neurosci 2016; 19:926-934.

13 Scott MM, Wylie CJ, Lerch JK, et al. Proc Natl Acad Sci USA 2005; 102:16472-16477.

14 Backman CM, Malik N, Zhang Y, et al. Genesis 2006; 44:383-390.

(Supplementary information is linked to the online version of the paper on the Cell Research website.) 